\title{
Supply Chain Model on Uncertainty Demand
}

\author{
Dewi Gusti ${ }^{1}$, Lolyta Damora Simbolon ${ }^{2}$, Yuan Anisa ${ }^{3}$, Liza Setyaning Pertiwi ${ }^{4}$ \\ ${ }^{1}$ University of North Sumatra, Department of Mathematics, Bioteknologi Road, Medan, Indonesia, SMKN 1 STABAT \\ 2, 3. ${ }^{4}$ University of North Sumatra, Department of Mathematics, Bioteknologi Road, Medan, Indonesia
}

\begin{abstract}
The life cycle of new product is becoming shorter and shorter in all markets. Given these short product life cycles, product demand is increasingly difficult to forecast. Furthermore, demand is never really stationary because the demand rates evolves over the life of the product. In this paper, we consider the problem of where in supply chain to place strategic safety stocks to provide a high level service to the final customer with minimum cost, and extend the model for stationary demand to the case of stationary demand, as might occur for products with short life cycles. We assume that we can model the supply chain as a network that each stage in the supply chain operates with a periodic reviuw base-stock policy, that demand is bounded and that there is guaranteed service time between every stage and its customers. This study uses the analytic solver program in excel that does the randomization process on the variable demand.
\end{abstract}

Keywords: nonstationary demand, multistage supply chain application, safety stock optimization.

\section{Introduction}

Manufacturing firms are introducing new products at a higher frequency with increasingly shorter life cycles. For each new product, a firm must determine its supply chain and the associated operating policies to match supply to the demand to achieve the most revenue with the least cost. A major complication is that the demand process evolves over the product life cycle and is never stationary.

The product life cycle of a new product typically consists of four phases: (i) a product-launch phase in which the product is introduced to the market; (ii) a demand-ramp phase over which the demand rate grows rapidly; (iii) a peak-demand phase during which the product sells at its maximum rate; and (iv) an end-of-life phase during which the product demand declines as it is removed from the market.

The demand rate is never stationary because the product moves from one life-cycle phase to another. This research examines the problem of locating safety stocks in a supply chain in a way that accounts for uncertain, nonstationary demand processes. Given the inherent complexity of modeling nonstationary demand processes, we seek a pragmatic approach that requires approximations and compromises to get results that might apply in practice. We use the modeling framework from Graves and Willems (2000) (referenced as G-W) and introduce a nonstationary demand model. We show that the G-W safety stock placement optimization applies to this case of nonstationary demand.

In the remainder of this section, we briefly discuss related literature. In $\S 2$, we present the key assumptions for modeling a supply chain and its nonstationary demand. In $\S 3$, we extend the G-W model to accommodate nonstationary demand. In $\S 4$, we examine a simple example to explore the near optimality of a constant service time (CST) policy.

Related Literature: Relative to the stationary-demand inventory literature, there is much less work for nonstationary demand. We characterize this work by how the nonstationary demand is specified and whether the work focuses on optimization versus performance evaluation. Morton and Pentico (1995) and Bollapragada and Morton (1999) focus on setting inventory policies for a single stage facing a general nonstationary demand process with proportional holding and backorder costs. When the order cost is zero, a time-varying base-stock policy is optimal; for a nonzero order cost, a time-varying $S_{-} S_{-}$policy is optimal. This research develops computationally efficient upper and lower bounds on these optimal policies. For short life cycle products, the challenge of accurately forecasting demand can be as important as determining inventory policies. Kurawarwala and Matsuo (1996) develop an integrated framework for forecasting and inventory management of short lifecycle products. Their approach estimates the parameters for a seasonal trend growth model and uses this as an input to a finite-horizon stochastic inventory model with time-dependent demands.

Nonstationary demand has also been modeled as a Markovmodulated Poisson demand process. One example is Chen and Song (2001), who show the optimality of echelon basestock policies with statedependent order-up-to levels for serial networks. A second example is Abhyankar and Graves (2001) who determine the optimal position of an inventory hedge in a two-stage serial supply chain that faces Markovmodulated demand with two states. Within the bullwhip literature, several papers develop models for nonstationary demand.

Papers generally assume that each stage follows an adaptive base-stock policy and then analyze the effect that different forecasting techniques and assumed demand distributions have on the inventory requirements at each stage. For instance, Lee et al. (1997) demonstrate that the adjustment of order-up-to levels at the retailer amplifies the variance of the order signal the retailer provides for the manufacturer. Two other examples are Graves (1999) and Chen et al. (2000).

Finally, there is a growing body of work on designing supply chains to handle nonstationary demand. Beyer and Ward

\section{Volume 4 Issue 11, November 2015}




\section{International Journal of Science and Research (IJSR) \\ ISSN (Online): 2319-7064}

Index Copernicus Value (2013): 6.14 | Impact Factor (2014): 5.611

(2000) use simulation to accurately model the inventory requirements in a two-echelon supply chain that utilizes two modes of distribution and is subject to nonstationary demand. Johnson and Anderson (2000) investigate the benefits of postponement in supply chains that introduce multiple products with short product life cycles. Ettl et al. (2000) minimize the total inventory in a multistage inventory system, where the key challenge is to approximate the replenishment lead times within the supply chain. To model nonstationary demand, they break the horizon into a set of stationary phases and adopt a rolling-horizon approach where the optimization is performed for each demand phase.

\section{Supply Chain Model}

In this section, we present the multistage model of a supply chain and the optimization problem for determining safety stocks.

Inventory Model. The single-stage inventory model serves as the building block for modeling a multistage supply chain. We adapt the standard model of Kimball (1988) (see also Simpson 1958) to the case of nonstationary demand.

We define the inbound service time SI, as the time for stage $\mathrm{j}$ to get suppliesfrom its immediate suppliers. In each period t, stage $\mathrm{j}$ places an order equal to $\phi_{\mathrm{ij}} \mathrm{d}_{\mathrm{j}}(\mathrm{t})$ on each upstream stage $\mathrm{i}$ for which $\phi_{\mathrm{ij}}>0$. The time for all orders to be delivered to stage $\mathrm{j}$ dictates when stage $\mathrm{j}$ can commence production to replenish its demand. This inbound service time is constrained by the maximum outbound service time from the upstream suppliers, i.e, $\mathrm{SI}_{\mathrm{ij}} \geq \max _{(\mathrm{i}, \mathrm{j}) \in \mathrm{A}}\left(\mathrm{S}_{\mathrm{i})}\right.$.

We assume we are given base stocks $B_{j}(t)$ for each period $t=1,2, \ldots, H$. For the stated assumptions, we can express the inventory at stage $\mathrm{j}$ at the end of period $\mathrm{t}$ as

$$
I_{j}(t)=B_{j}(t)-d_{j}\left(t-S I_{j}-T_{j}, t-S_{j}\right),
$$

where $d_{i}(s, t)=0$ for $s \leq t \leq 0$ and $d_{i}(s, t)=d_{i}(0, t)$ for $s<0<t$.

We defined the net replenishment time for stage $\mathrm{j}$ to be its replenishment time, net the stage's promised outbound service time, i.e., $\mathrm{SI}_{\mathrm{j}}+\mathrm{T}_{\mathrm{j}}-\mathrm{S}_{\mathrm{j}}$. This net replenishment time determines the safety stock at stage $\mathrm{j}$. We always set the outbound service times so that the net replenishment time is nonnegative.

The explanation for Equation (2) follows that for the case stationary or certainty demand. There are three transactions in period $t$ :stage $\mathrm{j}$ completes the replenishment of its demand from period $t-\mathrm{SI}_{\mathrm{j}}-\mathrm{T}_{\mathrm{j}}$; stage $\mathrm{j}$ fills its demand from period $\mathrm{t}-\mathrm{S}_{\mathrm{j}}$; and stage $\mathrm{j}$ receives an additional replenishment equal to $\Delta B_{j}(t)=B_{j}(t)-B_{j}(t-1)$ so as to have the prescribed base-stock level. Hence, we can write an inventory balance equation:

$$
I_{j}(t)=I_{j}(t-1)+d_{j}\left(t-S I_{j}-T_{j}\right)-d_{j}\left(t-S_{j}\right)+\Delta B_{j}(t) .
$$

We obtain (2) by applying (3) recursively and using the boundary condition $\mathrm{I}_{\mathrm{j}}(0)=\mathrm{B}_{\mathrm{j}}(0)$.

To derive Equation (2), we implicitly assume that we can always make the necessary adjustment $\Delta \mathrm{B}_{\mathrm{j}}(\mathrm{t})$ to the base- stock level. This need not be the case when the base-stock level decreases and $\Delta \mathrm{B}_{\mathrm{j}}(\mathrm{t})<0$; in effect, we need to assume that $\mathrm{d}_{\mathrm{j}}\left(\mathrm{t}-\mathrm{SI}_{\mathrm{j}}-\mathrm{T}\right)+\Delta \mathrm{B}_{\mathrm{j}}(\mathrm{t}) \geq 0$ so that the replenishment in period $t$ is nonnegative. We expect this will typically be the case, and we assume this to be true so as not to overly complicate the presentation. (We note that when $\mathrm{d}_{\mathrm{j}}\left(\mathrm{t}-\mathrm{SI}_{\mathrm{j}}-\mathrm{T}\right)+\Delta \mathrm{B}_{\mathrm{j}}(\mathrm{t})<0$, then Equation (2) provides a lower bound on the actual inventory level.)

Determination of Base Stock. For stage $j$ to provide 100\% service to its customers, we require that $I_{j}(t) \geq 0$; we see from Equation (2) that this requirement equates to

$$
B_{j}(t) \geq d_{j}\left(t-S I_{j}-T_{j}, t-S_{i}\right) \text {. }
$$

Because demand is bounded, we satisfy the above requirement with the least inventory by setting the base stock as

$$
B_{j}(t)=D_{j}\left(t-S I_{j}-T_{j}, t-S_{j}\right) .
$$

Thus, the base-stock level in period $t$ is the maximum possible demand over a time interval $\left(t-S I_{j}-T_{j_{-}}, t-S_{j}\right)$ for which stage $j$ filled its demand, but has yet to receive replenishments.

Safety Stock Model. We use Equations (2) and (4) to find the expected inventory level $E\left[I_{i}(t)\right]$ :

$$
\begin{aligned}
E\left[I_{j}(t)\right] & =D_{j}\left(t-S I_{j}-T_{j}, t-S_{j}\right)-\int_{\tau=t-S I_{j}-T}^{t-S_{j}} \mu_{j}(\tau) d \tau \\
& =g_{j}\left(t-S I_{j}-T_{j}, t-S_{j}\right) .
\end{aligned}
$$

The expected inventory represents the safety stock held at stage $j$ and depends on the net replenishment time and the demand bound. We observe that stage $j$ holds no safety stock whenever the net replenishment time is zero, i.e., $S I_{j}+T_{j^{-}}$ $S_{j}=0$.

The supply chain will also have a work-in-process or pipeline inventory. This inventory corresponds to the replenishment of customer demand plus the planned adjustments to the base-stock levels. If we fix the base-stock levels for the start and end of the planning horizon, then we can show that the work in process does not depend on the choice of service times, but only on the average demand rates and the lead times at each stage. Hence, in posing an optimization problem, we ignore work-in-process and only model safety stock.

Multistage Model. To model the multistage system, we use Equation (5) for each stage where inbound service time is a function of the outbound service times for the upstream stages. We then formulate an optimization problem to find the optimal service times for the planning horizon:

$$
\begin{aligned}
\mathbf{P} \text { Min } & \sum_{t=1}^{H} \sum_{j=1}^{N} h_{j} E\left[I_{j}(t)\right]=\sum_{t=1}^{H} \sum_{j=1}^{N} h_{j} g_{j}\left(t-S I_{j}-T_{j}, t-S_{j}\right) \\
\text { subject to } & S_{j}-S I_{j} \leq T_{j} \quad \text { for } j=1, \ldots, N \\
& S I_{j}-S_{i} \geq 0 \quad \forall(i, j) \in A \\
& S_{j}=0 \quad \forall \text { demand nodes } j \\
& S_{j}, S I_{j} \geq 0, \text { integer for } j=1, \ldots, N,
\end{aligned}
$$

where $h j$ denotes the holding cost per unit per time period for inventory at stage $j$. The objective of problem $\mathrm{P}$ is to minimize the safety stock holding cost 


\section{International Journal of Science and Research (IJSR) \\ ISSN (Online): 2319-7064}

Index Copernicus Value (2013): 6.14 | Impact Factor (2014): 5.611

over the planning horizon. The constraints assure that the net replenishment times are nonnegative, that each stage's inbound service time is no less than the maximum outbound service time quoted to the stage, and that the end-item stages satisfy their service guarantee. 3

We define the planning model to end in period $H$ and do not explicitly include any costs beyond this horizon. For instance, if $H$ represents the end of the life cycle for a product, there would be disposal costs for the supply chain inventory left over at the end of the horizon. We could include a disposal cost by restating $\mathrm{P}$ with time-dependent holding costs.

To solve P, we first observe that we can rewrite the objective function as

$$
\sum_{t=1}^{H} \sum_{j=1}^{N} h_{j} g_{j}\left(t-S I_{j}-T_{j}, t-S_{j}\right)=H \times \sum_{j=1}^{N} h_{j} G_{j}\left(S I_{j}, S_{j}\right) \text {, }
$$

where $G_{j}\left(S I_{j}, S_{j}\right)=H^{-1} \times \sum_{t=1}^{H} g_{j}\left(t-S I_{j}-T_{j}, t-S_{j}\right) \quad$ is the average safety stock at node $j$ as a function of its inbound and outbound service times. Thus, $\mathrm{P}$ is equivalent to the safety stock optimization problem for stationary demand in G-W, but with its objective function expressed in terms of the average safety stock $G_{j}\left(S I_{j}, S_{j}\right)$ over the planning horizon $H$. Furthermore, $G_{j}\left(S I_{j}, S_{j}\right)$ is a concave function, given the assumption that the net demand bound $g_{j}(s, t)$ is a concave function. As a consequence, we can solve $\mathrm{P}$ with the existing algorithms for stationary demand. G-W presents a dynamic programming algorithm for solving $\mathrm{P}$ for supply chains modeled as spanning trees; Humair and Willems (2006), Magnanti et al. (2006), and Lesnaia (2004) have each developed and tested algorithms for general acyclic networks.

\section{Example}

Ben is a purchasing agent for a large grocery store in charge of a new brand of canned pasta. His task is to make sure he always has product on hand to sell. However, due to limited storage space, any product being stored is subject to a $\$ 0.25$ holding cost. The lead time for an order is two weeks and each time an order is placed, there is a $\$ 75$ shipping fee. No more than 1,000 cans of product can be ordered at one time. The cost associated with a customer coming to the store and not being abe to purchase the product is $\$ 125$ in lost sales since the customer could decide to visit another grocer and not return. The demand for the product follows a Poisson distribution. Ben has created a simulation optimization model to determine at what point he should reorder the product (Reorder point) and how much to order (Order Quantity) to minimize total costs.

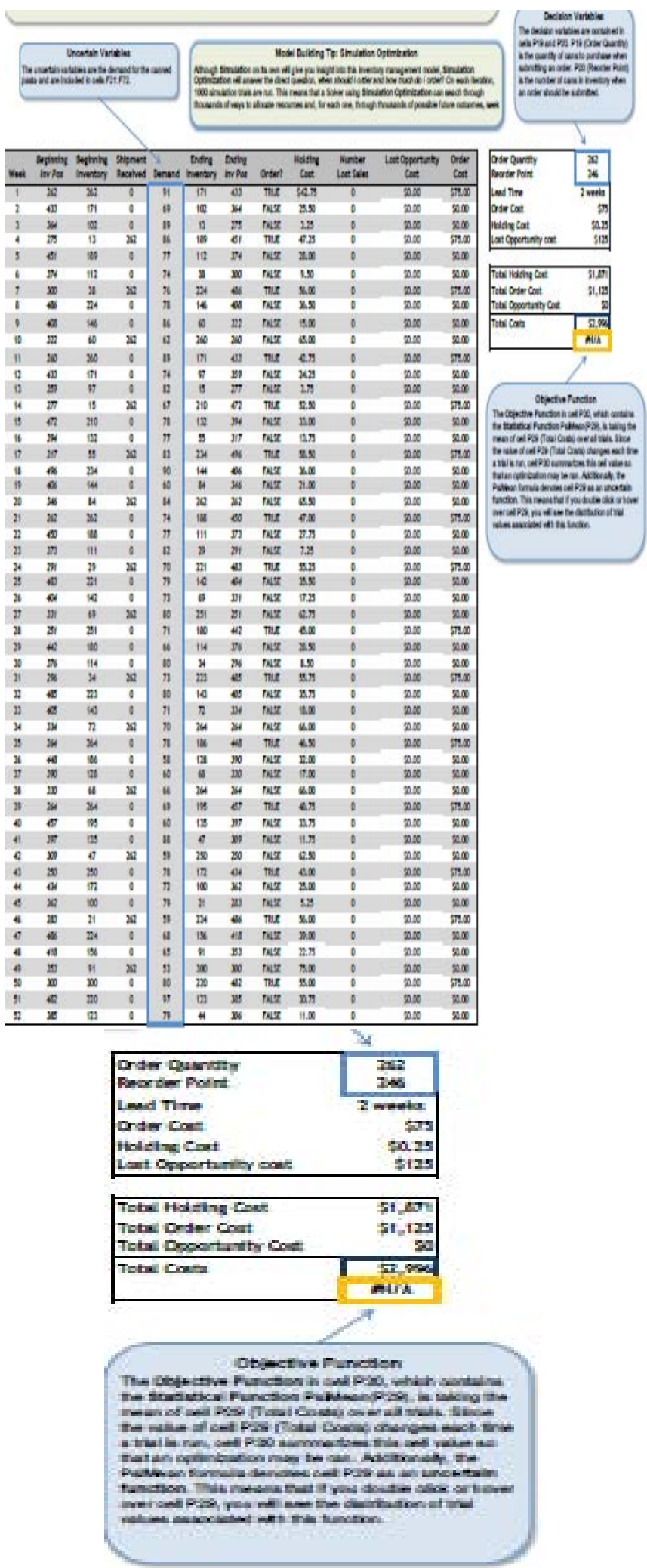

In this study, the model function represents the difference between the total inventory stock subject to the demand indicated by:

$$
\begin{gathered}
I j(t)=B j(t)-d j(t-S I j-T j, t-S j), \text { dimana } \\
B j(t)=\text { begin inventory }+ \text { shipment received }
\end{gathered}
$$

Stage 1 (based on data randomization of request simulation $1)$ :

$$
\begin{gathered}
\mathrm{Bj}(\mathrm{t})=\text { begin inventory }+ \text { shipment received } \\
\mathrm{Bj}(\mathrm{t})=262+0=262
\end{gathered}
$$




\section{International Journal of Science and Research (IJSR) \\ ISSN (Online): 2319-7064}

Index Copernicus Value (2013): 6.14 | Impact Factor (2014): 5.611

$\operatorname{Ij}(\mathrm{t})=\mathrm{Bj}(\mathrm{t})-\operatorname{dj}(\mathrm{t}-\mathrm{SIj}-\mathrm{Tj}, \mathrm{t}-\mathrm{Sj})=$ ending inventory $\operatorname{Ij}(\mathrm{t})=262-68=194$

Thus obtained function model to minimize the total cost:

$$
\text { Total holding cost }=\min \sum_{i=1}^{H} \sum_{j=1}^{N} h_{j} E\left[I_{j}(t)\right]
$$

Total holding cost $=0.25$ dollar $\times$ ending inventory

Total holding cost $=0.25$ dollar $\times 194=48.50$ dollar

Stage 2 (based on data randomization of request simulation 1):

$\operatorname{Bj}(\mathrm{t})=$ begin inventory + shipment received

$\operatorname{Bj}(\mathrm{t})=194+0=194$

Where, begin inventory $=194$ is data obtained at stage 1 .

$\operatorname{Ij}(t)=\operatorname{Bj}(t)-\operatorname{dj}(t-S I j-T j, t-S j)=$ ending inventory

$\operatorname{Ij}(\mathrm{t})=194-74=120$

Thus obtained function model to minimize the total cost:

$$
\text { Total holding cost }=\min \sum_{t=1}^{H} \sum_{j=1}^{N} h_{j} E\left[I_{j}(t)\right]
$$

Total holding cost $=0.25$ dollar $\times$ ending inventory

Total holding cost $=0.25$ dollar $\times 120=48.50$ dollar

The next stage is done in the same manner in which the stage $\mathrm{j}$ begin using the data inventory at stage $\mathrm{j}-1$,

Total cost $=$ total holding cost + total order cost

Total cost $=1.972$ dollar +1.125 dollar $=3.097$ dollar

Example 2: program on the attachment (simulation 2) Stage 1 (based on data randomization of request simulation 1):

$\mathrm{Bj}(\mathrm{t})=$ begin inventory + shipment received

$\operatorname{Bj}(\mathrm{t})=262+0=262$

$\operatorname{Ij}(t)=B j(t)-\operatorname{dj}(t-S I j-T j, t-S j)=$ ending inventory

$\operatorname{Ij}(t)=262-70=192$

Thus obtained function model to minimize the total cost:

$$
\text { Total holding cost }=\min \sum_{i=1}^{H} \sum_{j=1}^{N} h_{j} E\left[I_{j}(t)\right]
$$

Total holding cost $=0.25$ dollar $\times$ ending inventory

Total holding cost $=0.25$ dollar $\times 192=48$ dollar

Stage 2 (based on data randomization of request simulation 1):

$\mathrm{Bj}(\mathrm{t})=$ begin inventory + shipment received

$\operatorname{Bj}(\mathrm{t})=192+0=194$

which begin inventory $=194$ is data obtained at stage 1 ,

$\operatorname{Ij}(t)=\operatorname{Bj}(t)-\operatorname{dj}(t-S I j-T j, t-S j)=$ ending inventory

$\operatorname{Ij}(t)=194-68=124$

Thus obtained function model to minimize the total cost:

Total holding cost $=\min \sum_{t=1}^{H} \sum_{j=1}^{N} h_{j} E\left[I_{j}(t)\right]$

Total holding cost $=0.25$ dollar $\times$ ending inventory

Total holding cost $=0.25$ dollar $\times 124=31$ dollar
The next stage is done in the same manner in which the stage $\mathrm{j}$ begin using the data inventory at stage $\mathrm{j}-1$,

\section{Conclusion}

In this study, has been introduced and developed a model of placement safety stock in a supply chain with stationary demand and demonstrated how to extend supply chain model with demand stationary to obtain optimal safety stock placement in particular in short distribution products.

From ten simulations that have been done in this study, it was found the total cost is minimal, namely the simulation 4th with a total cost of 3,035 dollar, which amounted to 3981 units total demand and total supply of 7339 units. Means the initial stock of goods stored the remainder of last year by $7339-3981=3358$ units. The total cost of storage for 1,835 dollars and the total cost booking of 1,200 dollars. The more simulations performed so possible total cost of the smaller will be obtained. It relies on randomization demand, initial stock, and receipt of goods.

\section{References}

[1] Abhyankar, H. S., S. C. Graves. 2001. Creating an inventory hedge for Markov-modulated Poisson demand: An application and a model. Manufacturing Service Oper. Management 3 306-320.

[2] Beyer, D., J. Ward. 2000. Network server supply chain at HP: A case study. HP Labs Technical Report HPL2000-84, Hewlett Packard, Palo Alto, CA.

[3] Bollapragada, S., T. E. Morton. 1999. A simple heuristic for computing nonstationary $s_{-} S_{-}$policies. Oper. Res. 47 576-584.

[4] Chen, F., J.-S. Song. 2001. Optimal policies for multiechelon inventory problems with Markovmodulated demand. Oper. Res. 49 226-234.

[5] Chen, F. Y., J. K. Ryan, D. Simchi-Levi. 2000. The impact of exponential smoothing forecasts on the bullwhip effect. Naval Res. Logist. 47 269-286.

[6] Coughlin, R. L. 1998. Optimization and measurement of a worldwide supply chain. S.M. thesis, Leaders for Manufacturing Program, MIT, Cambridge, MA.

[7] Ettl, M., G. E. Feigin, G. Y. Lin, D. D. Yao. 2000. A supply network model with base-stock control and service requirements. Oper. Res. 48 216-232.

[8] Graves, S. C. 1999. A single-item inventory model for a nonstationary demand process. Manufacturing Service Oper. Management 1 50-61.

[9] Graves, S. C., S. P. Willems. 1998. Optimizing strategic safety stock placement in supply chains. Working paper, http://web.mit.edu/sgraves/www/papers/.

[10] Graves, S. C., S. P. Willems. 2000. Optimizing strategic safety stock placement in supply chains. Manufacturing Service Oper. Management 2 68-83.

[11] Graves, S. C., S. P. Willems. 2002. Strategic inventory placement in supply chains: Nonstationary demand. Working paper, http://web.mit.edu/sgraves/www/papers/. 
[12]Humair, S., S. P. Willems. 2006. Optimal inventory placement in networks with clusters of commonality. Oper. Res. 54 725-742.

[13] Johnson, M. E., E. Anderson. 2000. Postponement strategies for channel derivatives. Internat. J. Logist. Management 11 19-35.

[14] Kimball, G. E. 1988. General principles of inventory control. J. Manufacturing Oper. Management 1 119130.

[15] Kurawarwala, A., H. Matsuo. 1996. Forecasting and inventory management of short life-cycle products. Oper. Res. 44 131-150.

[16]Lee, H. L., V. Padmanabhan, S. Whang. 1997. Information distortion in a supply chain: The bullwhip effect. Management Sci. 43 546-558.

[17] Lesnaia, E. 2004. Optimizing safety stock placement in general network supply chains. PhD thesis, MIT Operations Research Center, Cambridge, MA.

[18] Magnanti, T. L., Z.-J. M. Shen, J. Shu, D. Simchi-Levi, C.-P. Teo. 2006. Inventory placement in acyclic supply chain networks. Oper. Res. Lett. 34 228-238.

[19] Morton, T. E., D. W. Pentico. 1995. The finite horizon nonstationary stochastic inventory problem: Nearmyopic bounds, heuristics, testing. Management Sci. 41 334-343.

[20] Simpson, K. F. 1958. In-process inventories. Oper. Res. $6863-873$. 